\title{
Systematic Adaptation of Exploration Force to Exploration Duration in Softness Discrimination
}

\author{
Aaron C. Zoeller ${ }^{(\bowtie)}$ and Knut Drewing \\ Giessen University, 35394 Gießen, Germany \\ aaron.zoeller@psychol.uni-giessen.de
}

\begin{abstract}
When interacting haptically with objects, humans enhance their perception by using prior information to adapt their behavior. When discriminating the softness of objects, humans use higher initial peak forces when expecting harder objects or a smaller difference between the two objects, which increases differential sensitivity. Here we investigated if prior information about constraints in exploration duration yields behavioral adaptation as well. When exploring freely, humans use successive indentations to gather sufficient sensory information about softness. When constraining the number of indentations, also sensory input is limited. We hypothesize that humans compensate limited input in short explorations by using higher initial peak forces. In two experiments, participants performed a 2 Interval Forced Choice task discriminating the softness of two rubber stimuli out of one compliance category (hard, soft). Trials of different compliance categories were presented in blocks containing only trials of one category or in randomly mixed blocks (category expected vs. not expected). Exploration was limited to one vs. five indentations per stimulus (Exp. 1), or to one vs. a freely chosen number of indentations (Exp. 2). Initial peak forces were higher when indenting stimuli only once. We did not find a difference in initial peak forces when expecting hard vs. soft stimuli. We conclude that humans trade off different ways to gather sufficient sensory information for perceptual tasks, integrating prior information to enhance performance.
\end{abstract}

Keywords: Perception $\cdot$ Softness $\cdot$ Exploration duration $\cdot$ Prior information

\section{Introduction}

Humans use their hands to explore the softness of objects every day. For example when squeezing a stress ball during work, when testing if bread is still good to eat or when palpating the leg to see if it is swollen. We use our hands rather than other senses, because softness is best explored by haptic interaction [1]. To explore softness, humans indent the surface of an object repeatedly to collect sensory information over time

This work was supported by Deutsche Forschungsgemeinschaft (DFG, German Research Foundation) - project number 222641018 - SFB/TRR 135, A5.

(C) The Author(s) 2020

I. Nisky et al. (Eds.): EuroHaptics 2020, LNCS 12272, pp. 105-112, 2020.

https://doi.org/10.1007/978-3-030-58147-3_12 
$[1,2]$. In everyday life the time during that objects can be explored may vary from free exploration to a highly constrained one (e.g., only one indentation). Here we ask how constraints in exploration duration influence softness exploration.

Perceived softness is not only, but clearly related to compliance [3]. The compliance of an object is defined as the amount of an object's deformation under a given force $[\mathrm{mm} / \mathrm{N}]$. In previous studies investigating softness perception using a 2 Alternative Forced Choice (2AFC) discrimination task paradigm, participants were reported to perform 3 to 7 indentations per stimulus, when allowed to explore freely [2, 4]. In free exploration, humans seem to stop exploring when they have sufficient information. With constant exploratory force, discrimination performance increases with the number of indentations up to a certain level. When exploration movements are restricted in duration, humans perform worse [2, 5].

However, perception relies on multiple information sources [6], and it is known that humans can use prior knowledge about object properties to adapt their exploratory behavior in haptic perception to the present task conditions [7, 8]. For example, for softness perception results from our lab showed that humans use more force in the initial indentations when they expect to discriminate two harder objects as compared to two softer objects $[2,4,9]$. Humans also use higher forces when expecting less difference between the compliance of the to-be discriminated objects. These adaptations seem to be well chosen, because more deformation of an object's surface and the exploring finger (e.g. by applying more force) improves sensory information on softness [9-11]. We wonder whether humans would also use higher force, when they know that they are constrained in their exploration duration, but free to adapt other movement parameters. High force might be a means to counterbalance lack of sensory information in shorter exploration. We hypothesize that participants explore with more force when planning a shorter exploration. In line with previous results, we also expect that participants apply more initial force when they expect harder as compared to softer stimuli [2]. Finally, we hypothesize that adaptations to compliance category are more relevant and hence also more pronounced for short explorations, where initial lack of information cannot be compensated for later.

In two experiments we used a 2 Interval Forced Choice (2IFC) softness discrimination task to investigate how initial peak forces vary in dependence of the exploration duration and prior information about object compliance. In a discrimination task we can control the success rate to be at an intermediate level. We expect that an intermediate level of success increases the effort that participants spend on the task (cf. Exp.2 in [9]). Further, stimuli were presented sequentially in a 2IFC task rather than in a 2AFC task so that participants did not perform uncontrolled switches between stimuli. Switches would have confounded an interpretation of the initial peak force by exploration duration. In each trial participants explored two objects from either a hard or soft category. Trials with harder and softer objects were presented in a blocked manner (only trials of soft or hard stimuli; prior information about compliance induced through recurring presentation) or randomly mixed (no prior information). In Experiment 1 participants had to indent in different blocks each stimulus once vs. five times. In Experiment 2 we compared one-indentation explorations to free explorations. 


\section{Methods}

\subsection{Participants}

Sixteen healthy students ( $N=8$ per experiment) from Giessen University participated (6 males, average age in years: 24, range: $18-30$ ). All participants were right-handed, reported no motor and cutaneous impairments, had normal or corrected-to normal vision, and were naive to the study's purpose. They were paid $8 € / \mathrm{h}$. Methods were approved by the local ethics committee LEK FB06 and in accordance with the 2013 Declaration of Helsinki. Participants gave written informed consent.

\subsection{Stimuli and Setup}

Participants sat on a custom-made visuo-haptic workbench containing a 24" 3D screen $(120 \mathrm{~Hz}, 1600 \times 900$ pixel), a force sensor (resolution $0.05 \mathrm{~N}$, temporal resolution $682 \mathrm{~Hz}$ ), to collect data of executed force, and a PHANToM 1.5A haptic force feedback device (spatial resolution: $0.03 \mathrm{~mm}$, temporal resolution: $1000 \mathrm{~Hz}$ ), to collect finger position data in a $38 \times 27 \times 20 \mathrm{~cm}^{3}$ workspace (Fig. 1). The right index finger of participants was connected to the PHANToM via a spherical magnetic adapter, allowing maximum freedom in finger movements and bare-finger exploration. We used stereo glasses (Nvidia 3D Vision 2) to present a 3D virtual scene. Finger position was displayed via a green sphere $(3 \mathrm{~mm})$ in the scene. During contact with the stimuli the sphere disappeared to give no feedback about finger position or stimulus deformation. Participants looked at the screen through a front surface mirror (viewing distance $40 \mathrm{~cm}$ ) aligned with the haptic scene to ensure a natural connection between haptic action and visual feedback. A chinrest was used to stabilize the heads of participants. Audio signals were given via headphones (Senheiser HD 280 Pro). All devices were connected to a PC; custom-made software controlled experiment and data collection.

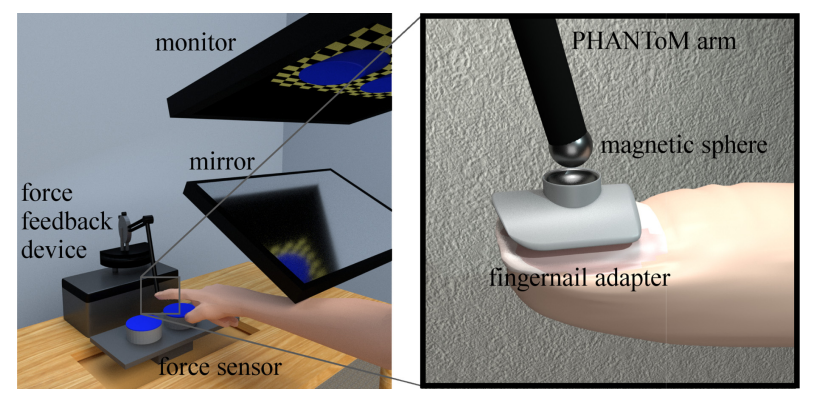

Fig. 1. Schema of the visuo-haptic workbench and the magnetic fingernail adapter.

In both experiments we used six cylindrical shaped silicone rubber stimuli (height: $38 \mathrm{~mm}$, diameter: $75 \mathrm{~mm}$; [9] for details of production). Stimuli were divided in a hard and a soft category. We used one stimulus per category as standard (hard: $0.16 \mathrm{~mm} / \mathrm{N}$; soft: $0.84 \mathrm{~mm} / \mathrm{N}$ ) and two stimuli per category as comparison stimuli (hard: 
$0.15 \mathrm{~mm} / \mathrm{N}$ and $0.17 \mathrm{~mm} / \mathrm{N}$; soft: $0.79 \mathrm{~mm} / \mathrm{N}$ and $0.88 \mathrm{~mm} / \mathrm{N})$. Stimuli were selected in a pilot study $(\mathrm{N}=8)$ based on their distinctness. Comparison stimuli were equally distinguishable (about $80 \%$ in free exploration) from the corresponding standard stimulus.

\subsection{Procedure and Design}

In both experiments we used a 2IFC softness discrimination task. We investigated the influence of three within-participant variables. Compliance $(C)$ : We presented stimuli from the soft or the hard compliance category. PriorCompliance $(P C)$ : In blocked conditions prior information about stimulus compliance was induced by presenting trials with stimuli out of one compliance category only. In randomized conditions, trials with stimuli out of the hard or soft category were presented in a randomly mixed order (no prior information about compliance). ExplorationDuration (ED): In Experiment 1, participants were instructed to indent each stimulus one or five times per trial (short vs. long exploration). In Experiment 2, participants indented each stimulus once or as often as they liked (short vs. free exploration). As can be seen in Fig. 1, the setup allowed participants to use their entire arm for force production. We measured initial peak forces, and perceptual performance as percentage of correct judgments.

In each trial, participants had to discriminate the softness of two stimuli (one standard and one comparison stimulus) and judge which one is softer. In the beginning one of the two stimuli was presented visually, to indicate where participants should start haptic exploration. Both starting positions were chosen equally often in randomized order. An acoustic signal indicated the start of the exploration. After participants indented the first stimulus as often as instructed (short exploration, long exploration and free exploration) it vanished from the visual scene and the second stimulus appeared on the screen. After indenting the second stimulus as often as instructed it vanished. By pressing one of two virtual buttons, participants indicated which of the two stimuli they had felt to be softer. No immediate feedback was given.

Both experiments consisted of two sessions within one week. In each session, participants conducted blocks of each combination of exploration duration and prior information conditions. Each combination overall comprised four blocks (two each day) consisting of 64 trials each (1024 trials in total). Between each block a break of one minute was implemented to counter fatigue. To counter sequence effects, half of the participants started with long explorations, the other half with short explorations on both days. We also balanced the order of blocks of randomized and blocked conditions within short and long/free exploration conditions on both days across participants, using $4 \times 4$ Latin squares. Each experiment took $6 \mathrm{~h}$ per participant.

\subsection{Data Analysis}

In this study we focused on analyzing initial peak forces in each trial, but also calculated the percentage of correct responses. Because initial peak forces are barely influenced by later sensory feedback, they are a good indicator of the influence of prior information on behavior [9]. In order to calculate peak forces, we initially subtracted stimulus mass from force measurements and smoothed the resulting force values over 
time, using a moving-averaging window with a kernel of $45 \mathrm{~ms}$. To capture peaks, we identified turning points in which the derivate of force over time changed from positive to negative. The applied force at this maximum additionally had to be higher than $5 \mathrm{~N}$. The time interval between two turning points was restricted to be at least $180 \mathrm{~ms}$. With these restrictions we ensured the exclusion of small finger shaking movements, local maxima and movement rests while releasing the finger from the object after valid peaks. Trials with more or less indentations than the allowed number (one, five or free exploration) were excluded from the analysis. The first captured maximum in each trial was assigned to be the initial peak force.

We compared initial peak forces (the first time indenting a stimulus in each trial) for all conditions in a repeated measurement ANOVA, using the three within-participant variables. In a further ANOVA with the same three variables we compared the percentage correct. Arcsine square root transformed percentages entered analysis, because these transforms approximate a normal distribution [12].

\section{Results}

For initial peak forces in Experiment 1, we found a significant main effect for ExplorationDuration $(E D), F(1,7)=27.44, p=.001$ indicating that participants used more force in shorter as compared to longer explorations (Fig. 2A). No other main effect or interaction was significant, Compliance $(C), F(1,7)=3.94, p=.087$; PriorCompliance $(P C): F(1,7)=0.02, p=.890 ; E D \times P C: F(1,7)=0.42, p=.539$; $E D \times C: F(1,7)=0.34, p=.580 ; P C \times C: F(1,7)=0.03, p=.859 ; E D \times P C \times C: F$ $(1,7)<0.01, p=.961$. We additionally investigated peak forces of the different indentations in the long exploration condition. For reasons of space, we cannot report each detail. An extra ANOVA with the variables Indentation $\left(1^{\text {st }}\right.$ to $\left.5^{\text {th }}\right)$, PriorCompliance, and Compliance, revealed no significant main effect and no interaction $(\alpha=5 \%)$. Five $t$-tests confirmed that peak force in each indentation of the long exploration condition (per indentation in $\mathrm{N}, 1^{\text {st }}: 17.3,2^{\text {nd }}: 17.6,3^{\text {rd }}: 17.6,4^{\text {th }}: 17.4,5^{\text {th }}$ : 18.8) was significantly lower than in the short exploration condition (each $p<.001$ ).

In Experiment 1, on average $67 \%$ of all answers were correct $(S E M=0.021$, range across individuals $63 \%-75 \%$ ). In the ANOVA we found a significant effect for the variable $P C, F(1,7)=10.09, p=.016$, indicating that participants performed better when prior information available (70\%) as compared to the randomized conditions $(65 \%)$. No other effects were significant $(\alpha=5 \%)$.

In Experiment 2, participants indented each stimulus on average 2.9 times in the free exploration condition. For initial peak forces (Fig. 2B), we again found a significant main effect for the variable $E D, F(1,7)=12.85, p=.009$. Other effects were not significant, $C: F(1,7)=0.02, p=.896 ; P C: F(1,7)=5.55, p=.051 ; E D \times P C$ : $F(1,7)=0.46, p=.518 ; E D \times C: F(1,7)=1.13, p=.322 ; P C \times C: F(1,7)=0.02$, $p=.896 ; E D \times P C \times C: F(1,7)=2.64, p=.148$. Extra analyses of peak forces of later indentations in the free exploration conditions showed some variation (range of averages about 16 to $19 \mathrm{~N}$ ), but for each indentation in each free exploration condition peak force was lower than in the corresponding short exploration condition (16 tests, each $p<.05)$. 
On average, participants responded correctly in $70 \%$ of all trials $(S E M=0.017$, range $67 \%$ to $73 \%$ ). In the ANOVA comparing performance, we again found a significant effect for the variable $P C, F(1,7)=21.78, p=.002$, indicating that participants performed better in blocked conditions $(73 \%)$ as compared to randomized conditions $(67 \%)$. We found no other significant effect $(\alpha=5 \%)$.
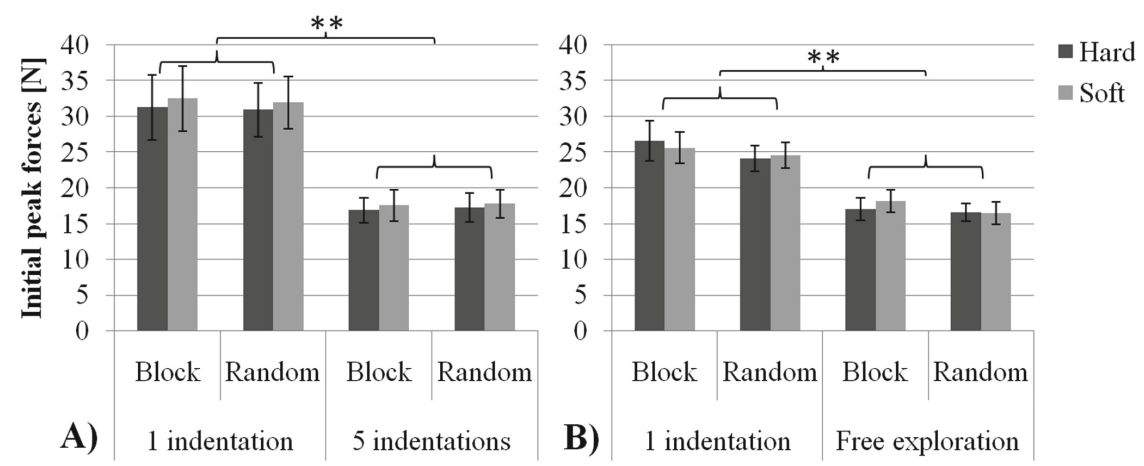

Fig. 2. Average initial peak forces $(\mathrm{N})$ and standard error of mean $(S E M)$ for each of the variable combinations of Experiment $1(\mathbf{2 A})$ and $2(\mathbf{2 B})$. Significant effects are marked $p<.01=* *$.

\section{Discussion}

In two experiments, we compared initial peak forces for different exploration durations with and without prior information about stimulus compliance. In contrast to previous studies $[2,4,9]$, we did not find adaptation of initial peak forces to predictable stimulus compliances. However, in both experiments we found a strong effect for the exploration duration indicating that participants used much more initial peak force when indenting stimuli only once as compared to five times or to free exploration (3 indentations, on average). Also, peak forces of following indentations were lower as compared to the initial peak force of the short exploration condition, indicating no compensation for low initial forces in later indentations. In line with [9], it seems that humans adapt their exploration forces when expecting shorter explorations, which do not allow for repetitive gathering of sensory information. It has been speculated that with more deformation of the finger and an object's surface more sensory information on softness can be gathered [9-11], and it has been shown that higher force can improve differential sensitivity for softness up to a certain maximum (Exp. 3 in [9]). Hence, we speculate that high force has served in the present study as a means to counterbalance lack of sensory information in shorter exploration. Further, we did not find performance differences between longer/free and shorter explorations (which had been reported, when force was held constant $[2,5])$. We conclude that our participants traded off force and exploration duration to gather sufficient sensory information.

Unexpectedly, prior information about an object's compliance did not have the expected effect on initial peak force. Still, participants performed better when prior 
information about the stimulus compliance was available. This may indicate that although no motor adaptation based on the prior information about stimulus compliance was found, the information was integrated in perception, enhancing performance.

But why did participants not use prior information on compliance for motor adaptation? One possible reason is that force adaptation based on exploration duration was dominant and rendered further force adaptation based on an object's compliance unnecessary: When sensory information input was limited due to short exploration, humans might already have used very high force to gather as much sensory information as possible. This adaptation might overshadow any useful force adaption based on stimulus compliance in the form of a ceiling effect. In contrast, when forced to indent the stimuli five times each (long exploration), participants might have expected to gather sufficient sensory information anyway and used low forces to save energy.

Notice that the presently found peak forces were higher as compared to some previous studies [e.g., 11, 13, 14]. However, contrary to the previous studies, in which participants produced forces using their finger muscles only, in the present setup participants used their entire arm. Present peak forces are comparable to those found in previous studies with the same setup [e.g. 2, 4, 9]. In [10], where participants were allowed to use their body to produce force, even higher peak forces between 80 and $400 \mathrm{~N}$ were found. Thus, the present peak forces do not seem exceptionally high.

However, the lack of adaptation during free exploration in Experiment 2 cannot be explained by this speculation. We speculate that the design of our study might have also interfered with natural adaptation behavior, as it is more constricted and less natural as compared to previously used designs, where participants were allowed to change back and forth between stimuli in order to compare them [2, 4, 9]. Here, movements were explicitly constrained to sequentially explore stimuli in each trial. As shown by Zoeller et al. [4], the appearance of peak force adaptation based on stimulus compliance might vanish with too explicit control of motor behavior.

Overall, we conclude that humans adapt their exploration behavior in softness discrimination to the expected exploration duration. When expecting a short exploration, humans use much more force to indent objects as compared to longer explorations. This tradeoff seems to be a sufficient strategy to enhance sensory perception. We suggest that humans trade off different ways to gather sufficient sensory information for perceptual tasks, integrating prior information to enhance performance. This, of course, has to be further tested in other perceptual domains.

\section{References}

1. Lederman, S.J., Klatzky, R.L.: Hand movements: a window into haptic object recognition. Cogn. Psychol. 19(3), 342-368 (1987)

2. Lezkan, A., Metzger, A., Drewing, K.: Active haptic exploration of softness: indentation force is systematically related to prediction, sensation and motivation. Front. Integr. Neurosci. 12, 59 (2018)

3. Bergmann Tiest, W.M., Kappers, A.M.L.: Physical aspects of softness perception. In: Di Luca, M. (ed.) Multisensory Softness. SSTHS, pp. 3-15. Springer, London (2014). https:// doi.org/10.1007/978-1-4471-6533-0_1 
4. Zoeller, A.C., Lezkan, A., Paulun, V., Fleming, R., Drewing, K.: Integration of prior knowledge during haptic exploration depends on information type. J. Vis. 19, 20 (2019)

5. Lezkan, A., Drewing, K.: Processing of haptic texture information over sequential exploration movements. Atten. Percept. Psychophys. 80(1), 177-192 (2017). https://doi. org/10.3758/s13414-017-1426-2

6. Ernst, M.O., Bülthoff, H.H.: Merging the senses into a robust percept. Trends Cogn. Sci. 8 (4), 162-169 (2004)

7. Lederman, S.J., Klatzky, R.L.: Haptic classification of common objects: knowledge-driven exploration. Cogn. Psychol. 22(4), 421-459 (1990)

8. Tanaka, Y., Bergmann Tiest, W.M., Kappers, A.M., Sano, A.: Contact force and scanning velocity during active roughness perception. PloS ONE 9(3), e93363 (2014)

9. Kaim, L., Drewing, K.: Exploratory strategies in haptic softness discrimination are tuned to achieve high levels of task performance. IEEE Trans. Haptics 4(4), 242-252 (2011)

10. Nicholson, L.L., Maher, C.G., Adams, R.: Hand contact area, force applied and early nonlinear stiffness (toe) in a manual stiffness discrimination task. Man. Ther. 3(4), 212-219 (1998)

11. Srinivasan, M.A., LaMotte, R.H.: Tactual discrimination of softness. J. Neurophysiol. 73(1), 88-101 (1995)

12. Claringbold, P.J., Biggers, J.D., Emmens, C.W.: The angular transformation in quantal analysis. Biometrics 9(4), 467-484 (1953)

13. Xu, C., Wang, Y., Hauser, S.C., Gerling, G.J.: In the tactile discrimination of compliance, perceptual cues in addition to contact area are required. In: Proceedings of Human Factors Ergonomics Society, vol 42, pp. 1535-1539. SAGE Publications, Los Angeles (2018)

14. Friedman, R.M., Hester, K.D., Green, B.G., LaMotte, R.H.: Magnitude estimation of softness. Exp. Brain Res. 191(2), 133-142 (2008). https://doi.org/10.1007/s00221-008$1507-5$

Open Access This chapter is licensed under the terms of the Creative Commons Attribution 4.0 International License (http://creativecommons.org/licenses/by/4.0/), which permits use, sharing, adaptation, distribution and reproduction in any medium or format, as long as you give appropriate credit to the original author(s) and the source, provide a link to the Creative Commons license and indicate if changes were made.

The images or other third party material in this chapter are included in the chapter's Creative Commons license, unless indicated otherwise in a credit line to the material. If material is not included in the chapter's Creative Commons license and your intended use is not permitted by statutory regulation or exceeds the permitted use, you will need to obtain permission directly from the copyright holder.

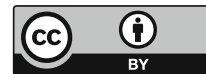

